\title{
Theoretical study on interaction of hydrogen with single-walled boron nitride nanotubes. II. Collision, storage, and adsorption
}

\author{
Sang Soo Han, Jeung Ku Kang, and Hyuck Mo Lee ${ }^{\text {a) }}$ \\ Department of Materials Science and Engineering, Korea Advanced Institute of Science and Technology \\ (KAIST), Daejeon 305-701, Republic of Korea
}

Adri C. T. van Duin and William A. Goddard III

Materials and Process Simulation Center, California Institute of Technology, California 91125

(Received 2 November 2004; accepted 21 June 2005; published online 19 September 2005)

\begin{abstract}
Collision and adsorption of hydrogen with high incident kinetic energies on a single-walled boron nitride (BN) nanotube have been investigated. Molecular-dynamics (MD) simulations indicate that at incident energies below $14 \mathrm{eV}$ hydrogen bounces off the BN nanotube wall. On the other hand, at incident energies between 14 and $22 \mathrm{eV}$ each hydrogen molecule is dissociated at the exterior wall to form two hydrogen atoms, but only one of them goes through the wall. However, at the incident energies between 23 and $26 \mathrm{eV}$ all of the hydrogen atoms dissociated at the exterior wall are found to be capable of going inside the nanotube and then to recombine to form hydrogen molecules inside the nanotube. Consequently, it is determined that hydrogen should have the incident energy $>22 \mathrm{eV}$ to go inside the nanotube. On the other hand, we find that the collisions using the incident energies $>26 \mathrm{eV}$ could result in damaging the nanotube structures. In addition our MD simulations find that hydrogen atoms dissociated at the wall cannot bind to either boron or nitrogen atoms in the interior wall of the nanotube. (C) 2005 American Institute of Physics.
\end{abstract}

[DOI: $10.1063 / 1.1999629]$

\section{INTRODUCTION}

The first experimental study of Dillon et al. ${ }^{1}$ on hydrogen storage in carbon nanotubes (CNTs) has triggered a worldwide tide of research on CNTs. On the other hand, several controversial results ${ }^{2-6}$ against this report have been reported so far. The additional issue is that most of previously used samples were too small to allow accurate determination of a true hydrogen storage capacity, thus it would be also true that some results of rather high storage capacities are attributed to experiment faults. Moreover, it should be noted that most of measured hydrogen storage capacities at room temperature are too small on pristine CNTs, thus incapable of being used for practical applications. Consequently, nowadays it is very crucial to find out a good hydrogen storage material to be capable of overcoming some problems from the CNT. In this respect, here we determine whether the pristine boron nitride nanotube (BNNT) similar to the CNT might be a good candidate for a practical hydrogen storage material or not.

Our previous study ${ }^{6}$ showed that the pristine CNT is not an effective hydrogen storage medium. This is attributed mainly to the small value for the enthalpy change in hydrogen adsorption on the CNT. For example, the change of heat for physisorption on the pristine CNTs of both single- and multiwalled types is in the range of about $1-3 \mathrm{kcal} / \mathrm{mol}$. Consequently, physisorption of hydrogen on the pristine CNT is considered to be only possible at low temperatures below $200 \mathrm{~K}$. On the other hand, once a hydrogen atom

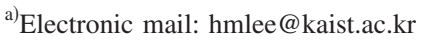

binds chemically to a carbon atom in the CNT wall, a high temperature should be required for making hydrogen atoms desorbed due to its high chemical binding energy. The structure for the BNNT is similar to that of the $\mathrm{CNT},{ }^{7,8}$ but its $\mathrm{B}-\mathrm{N}$ bond length of $1.46 \AA$ (Ref. 9) is larger than the $\mathrm{C}-\mathrm{C}$ bond length of $1.42 \AA$ in the CNT. Consequently, the BNNT is considered to have the faster rate for hydrogen penetration through the wall. In addition the recent studies ${ }^{10,11}$ showed that BN fullerenes have hydrogen storage capacities larger than carbon fullerenes. Moreover, Ma et al. ${ }^{12}$ reported that multiwalled bamboolike BNNT samples could store hydrogen up to $2.6 \mathrm{wt} \%$ at room temperature. Also Tang et al. ${ }^{13}$ discovered that BNNTs with a collapsed structure store up to hydrogen of $4.2 \mathrm{wt} \%$ at room temperature. For these reasons, the BNNT is considered as a better hydrogen storage medium than the CNT.

Narita and $\mathrm{Oku}^{10}$ studied $\mathrm{C}_{60}$ and $\mathrm{B}_{36} \mathrm{~N}_{36}$ clusters using first-principles calculations. They predicted the activation barriers for hydrogen penetration through tetragonal, pentagonal, and hexagonal rings, where the energies were determined at the varied positions of hydrogen at the interval of $0.1 \mathrm{~nm}$ from the center of the cage. To expand the electronic wave functions, the double-zeta Pople basis set of 3-21 G (Ref. 14) and the Hartree-Fock theory ${ }^{15}$ have been used. They reported that the activation barriers for hydrogen penetration through pentagonal and hexagonal rings of the $\mathrm{C}_{60}$ are 23 and $16 \mathrm{eV}$, respectively, while those through tetragonal and hexagonal rings of the $\mathrm{B}_{36} \mathrm{~N}_{36}$ are 27 and $14 \mathrm{eV}$, respectively. This indicates that hexagonal rings of $\mathrm{BN}$ fullerenes are better for hydrogen penetration than those of carbon fullerenes. On the other hand, it should be noted that 
the barriers of Narita and Oku were obtained by excluding zero-point corrections and thermal effects. Ma et al. ${ }^{16}$ reported the molecular-dynamics (MD) simulation results for collision of hydrogen atoms with the sidewall of a $(5,5)$ single-walled carbon nanotube (SWCNT) using various kinetic energies of the hydrogen atoms. These results include thermal effects. Ma et al. ${ }^{16}$ concluded that at least the incident energy of $16 \mathrm{eV}$ is required for a hydrogen atom to penetrate through the hexagons of the SWCNT.

We have not found yet any MD simulation study on collision and adsorption behaviors of hydrogen with high incident kinetic energies on the BNNTs. Here we explore collision and adsorption mechanisms of hydrogen with high incident energies on the BNNT using molecular-dynamics simulations. The MD simulations are conducted using a kinetic energy in the range of $1-35 \mathrm{eV}$. We also consider the effects of impingement locations of hydrogen. This work is to determine activation barriers for hydrogen penetration through the wall, and the behavior of hydrogen inside the nanotube after penetration. In addition we investigate adsorption properties by determining $\mathrm{H}$-adsorbed structures and their bonding energies.

\section{COMPUTATIONAL DETAILS}

To examine collision mechanisms of hydrogen on the single-walled BNNT (SWBNNT), hydrogen with the incident energies ranging from 1 to $35 \mathrm{eV}$ impinges onto the sidewall of a single-walled BNNT. The single-walled BNNTs were experimentally observed to have a range of diameters ranging from $\sim 0.5$ to $1.2 \mathrm{~nm} .^{17,18}$ In addition it was reported that synthesis of zigzag-type BNNTs is more favorable to that of the armchair type. ${ }^{8,19}$ Consequently, in this study we choose the $(10,0)$ zigzag single walled consisting of 60 boron and 60 nitrogen atoms with a diameter of $0.81 \mathrm{~nm}$. Since the SWBNNT has a very high aspect ratio, ${ }^{17,18}$ hydrogen would interact mostly with boron and nitrogen atoms in the wall of the nanotube. Therefore, our investigation focuses mainly on the hydrogen collision and adsorption mechanisms on the SWBNNT wall, rather than other hydrogen adsorption mechanisms such as that through diffusion into the open-ended tube. The interatomic potentials and forces among hydrogen, boron, and nitrogen are obtained using the reactive force field, $\mathrm{ReaxFF}_{\mathrm{HBN}}$, reported in the previous work, Part I. The MD simulations are performed under the canonical ensemble condition where the total atom number $(N)$, the temperature $(T)$, and the volume $(V)$ for the system are fixed. The simulation temperature is $300 \mathrm{~K}$, where the system under consideration is coupled to the heat bath which introduces energy variations needed to keep the temperature fixed. In addition the Verlet algorithm ${ }^{20}$ is employed to integrate equations of motion with a time step of $0.25 \mathrm{fs}$.

As illustrated in Fig. 1, the initial position for impingement on the nanotube by hydrogen is considered in four modes: (A) hydrogen is placed $3 \AA$ away from a hexagon of the tube wall, (B) hydrogen is placed $3 \AA$ from the hexagon at an angle of $45^{\circ}$ to the tube axis, (C) hydrogen is placed $3 \AA$ from a boron atom of the tube wall, and (D) hydrogen is
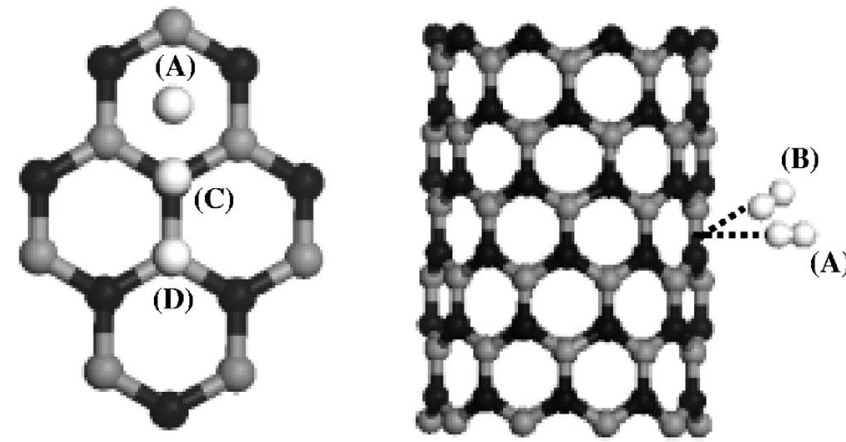

FIG. 1. Atomistic configurations of the initial positions of a hydrogen molecule: (A) perpendicular to a hexagon surface of a $(10,0)$ SWBNNT, (B) at an angle of $45^{\circ}$ to the tube axis, (C) perpendicular above a boron atom, and (D) perpendicular above a nitrogen atom. The black, gray, and white atoms denote boron, nitrogen, and hydrogen, respectively.

placed $3 \AA$ away from a nitrogen atom. In all the cases, hydrogen is set to move toward the sidewall with the velocity corresponding to the incident kinetic energy. All the impinging points in modes (A) and (B) are selected in the center of a hexagon of the sidewall. This hexagon is located in the middle part of the tube. The periodicity of a singlewalled BNNT ensures that this sampling represents any hexagon on the wall with an infinitely long tube shape. The impinging points in modes (C) and (D) are on the boron and nitrogen atoms, respectively. The positions for collision by the hydrogen atom are also obtained under similar conditions as the cases of the hydrogen molecule.

We also employ density-functional theory (DFT) to determine the binding energy for hydrogen adsorption on the wall of the nanotube. In this case, the structure model for calculations is based on the $(10,0)$ SWBNNT of a cluster type consisting of 40 boron and 40 nitrogen atoms. On the other hand, as the dangling bonds at both ends are tied off with hydrogen atoms, the true cluster model for DFT calculations is based on the $\mathrm{B}_{40} \mathrm{~N}_{40} \mathrm{H}_{20}$ structure. The DMOL ${ }^{3}$ program $^{21}$ was employed for full geometry optimizations, using the generalized gradient approximation (GGA) method that is parametrized by Perdew and Wang's scheme (PW91). ${ }^{22}$ The PW91 exchange-correlation functional was shown to predict well exchange and correlation energies of the first-row atoms. ${ }^{23}$ All the electronic wave functions are expanded in a local atomic-orbital basis. ${ }^{24}$ All the orbitals including those for core electrons are fully taken into account throughout the calculations. The unrestricted Hartree-Fock (UHF) method with a double-numerical basis set of DNP (Ref. 25) is also used to describe the polarization functions, equivalent to split-valence double-zeta plus polarization basis set.

\section{RESULTS AND DISCUSSION}

\section{A. MD simulation studies employing the ReaxFF $F_{\mathrm{HBN}}$}

In mode $(\mathrm{A})$ the collision process between a hydrogen molecule and a single-walled BNNT has been explored with various incident energies, and six different cases are observed: (i) the hydrogen molecule rebounds off the sidewall and the nanotube remains intact, (ii) one hydrogen atom pen- 


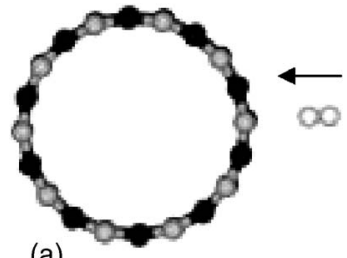

(a)

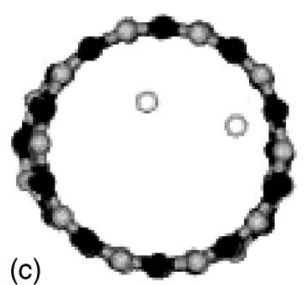

(c)

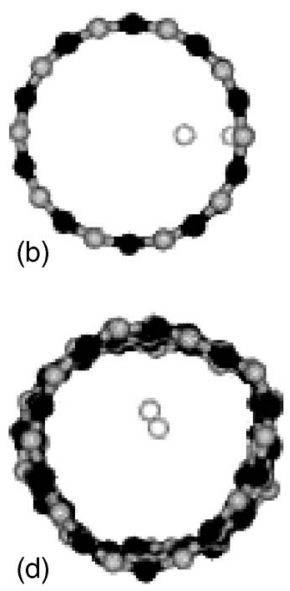

FIG. 2. Snapshots of interaction between a $(10,0)$ SWBNNT and a $\mathrm{H}_{2}$ molecule taken at simulation times of (a) 0, (b) 30, (c) 200, and (d) 950 steps when the incident energy of a $\mathrm{H}_{2}$ molecule is $26 \mathrm{eV}$.

etrates into the tube, then is trapped inside, while another hydrogen atom rebounds off the wall, (iii) both hydrogen atoms enter the tube and then trapped inside the tube, (iv) both hydrogen atoms enter the tube from one side and only one hydrogen atom escapes through the other side, (v) after both hydrogen atoms enter the tube from one side and escape through the other side, one hydrogen atom is chemically adsorbed on a boron atom on the exterior tube wall, which forms a hydrogenated single-walled nanotube (H-SWNT) complex, and finally (vi) both hydrogen atoms enter the tube from one side and escape through the other side.

It has been observed that case (i) takes place when the incident energy is in the range of 1 through $13 \mathrm{eV}$. The $\mathrm{H}_{2}$ molecule cannot penetrate through the BNNT wall. Instead, it rebounds off the sidewall and the nanotube remains intact, that is, any B-N bond of the tube is not broken. The nanotube is just distorted by collision.

In case (ii), a hydrogen molecule with the incident energy of $14-22 \mathrm{eV}$ is first dissociated into two hydrogen atoms after impingement. One hydrogen atom penetrates through the wall and another atom rebounds off the wall. After $500 \mathrm{MD}$ steps, the hydrogen atom trapped inside the tube wanders inside the nanotube. The $(10,0)$ SWCNT with the same helicity of the $(10,0)$ SWBNNT is also tested in terms of hydrogen penetration, and it is observed that hydrogen can enter more easily the BNNT than the CNT, in good agreement with previous DFT calculations. ${ }^{10,11}$ The difference in hydrogen penetration kinetics between the CNT and the BNNT is probably caused by a different hexagon size of the nanotube. The $\mathrm{B}-\mathrm{N}$ bond length $(1.46 \AA)$ in the SWBNNT is larger than the $\mathrm{C}-\mathrm{C}$ bond length $(1.42 \AA)$ in the SWCNT. Nonetheless, it is difficult for a hydrogen molecule to enter the SWBNNT because the smallest incident kinetic energy of the molecule for the process is $14 \mathrm{eV}$.

Case (iii) occurs in the energy range between 23 and $26 \mathrm{eV}$. Figure 2 shows the snapshot of interaction at an energy level of $26 \mathrm{eV}$. When a hydrogen molecule arrives at the center of the SWBNNT, it is first dissociated into two atoms, and then one hydrogen atom enters the tube wall,

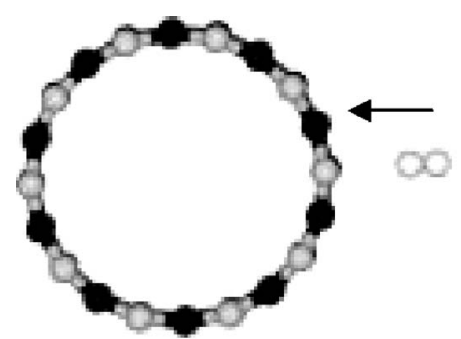

(a)

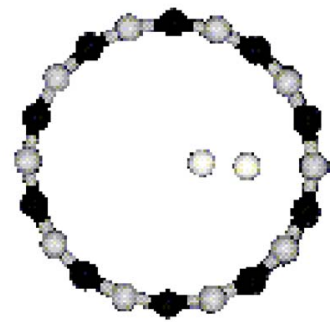

(b)

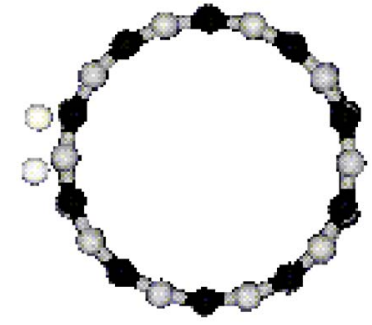

(c)

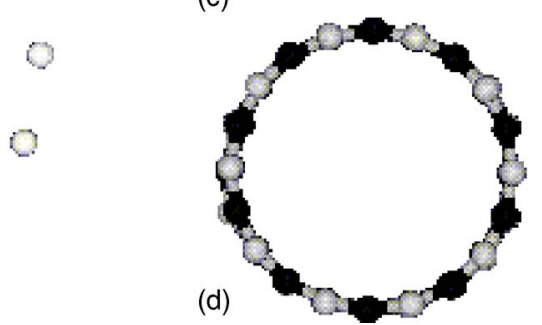

FIG. 3. Snapshots of interaction between a $(10,0)$ SWBNNT and a $\mathrm{H}_{2}$ molecule taken at simulation times of (a) 0 , (b) 30, (c) 70, and (d) 100 steps when the incident energy of a $\mathrm{H}_{2}$ molecule is $35 \mathrm{eV}$.

followed by another atom. As time increases, both of the hydrogen atoms wander inside the tube by van der Waals interaction with the tube wall, and then the two hydrogen atoms reform a hydrogen molecule in the tube.

In the energy range of $27-29 \mathrm{eV}$, case (iv), a hydrogen molecule is dissociated into two atoms on arrival, then one hydrogen atom enters the tube and another follows through. With increased time, the hydrogen atom that entered first escapes through the other side, while the other hydrogen atom is still captured by van der Waals interaction with the interior wall.

The favorable energy level for case (v) is $30 \mathrm{eV}$. Here, a $\mathrm{H}_{2}$ molecule is dissociated right after impingement, then one hydrogen atom enters the tube and another follows through. Due to the impact of hydrogen with a high kinetic energy, one $\mathrm{B}-\mathrm{N}$ bond in the tube is broken near the opposite sidewall, which results in the formation of a decagon which originated from two adjacent hexagons. Formation of the decagon causes a large hole for easy escaping of hydrogen. Eventually both hydrogen atoms escape through the decagon from the tube, however, one hydrogen atom is chemically bonded with a B atom having only two $\mathrm{B}-\mathrm{N}$ bonds in the decagon.

Finally above $31 \mathrm{eV}$ [case (vi)], a $\mathrm{H}_{2}$ molecule is first dissociated into two hydrogen atoms on arrival at the hexagon center, and then both hydrogen atoms enter the tube but escape through the other side, as seen in Fig. 3. No hydrogen atom or molecule remains inside the SWBNNT.

Up to now, all the simulations are conducted in mode 
TABLE I. Behaviors of a hydrogen molecule impinging with its incident direction and kinetic energy. Here modes (B), (C), and (D) are shown in Fig. 1.

\begin{tabular}{lcl}
\hline \hline & $\begin{array}{c}\text { Incident energy } \\
\text { range }(\mathrm{eV})\end{array}$ & \multicolumn{1}{c}{ Phenomena } \\
\hline Mode $(B)$ & $1-25$ & A hydrogen molecule rebounds off the sidewall of the SWBNNT. \\
& $26-35$ & Both hydrogen atoms rebound off the sidewall of the tube as atomic states. \\
Mode $(C)$ & $1-18$ & A hydrogen molecule rebounds off the sidewall of the tube. \\
& $19-35$ & Both hydrogen atoms rebound off the tube as atomic states. \\
Mode $(D)$ & $1-26$ & $\begin{array}{l}\text { A hydrogen molecule rebounds off the sidewall of the tube. } \\
\text { Both hydrogen atoms rebound off the tube as atomic states. }\end{array}$ \\
\hline \hline
\end{tabular}

(A). Behaviors of a hydrogen molecule for modes (B), (C), and (D) are summarized in Table I. In case (B), a hydrogen molecule rebounds off the sidewall of the $(10,0)$ SWBNNT in the energy range of $1-25 \mathrm{eV}$. Above $26 \mathrm{eV}$, when a hydrogen molecule arrives at the center of the nanotube, two hydrogen atoms temporarily bind with each $\mathrm{B}$ and $\mathrm{N}$ atoms near the impingement point, which results in hydrogen dissociation. Two hydrogen atoms rebound off the tube wall as atomic states. The nanotube remains intact in the case of mode (B) although it is impacted by a very high energy. If the impinging point of hydrogen is right on a vertex of boron [mode (C)] or nitrogen [mode (D)] atom, hydrogen rebounds off the sidewall, irrespective of the incident energy. Similar to mode (B), the hydrogen molecule rebounds off as a molecular state at a relatively low incident energy $[1-18 \mathrm{eV}$ for mode (C) and 1-26 for mode (D)], whereas it does as an atomic state at a high incident energy [above 19 and $27 \mathrm{eV}$, respectively, for modes (C) and (D)]. In mode $(C)$, if the incident energy of hydrogen is above $31 \mathrm{eV}$, one $\mathrm{B}-\mathrm{N}$ bond is broken near the boron atom impacted by hydrogen, which causes a decagon. However, the decagon is cured within a very fast time of about $1 \mathrm{ps}$ and then the impacted nanotube preserves its original structure. In mode (D), the nanotube always remains intact irrespective of incident energies of hydrogen. Based on the above results, it is difficult for a hydrogen molecule to penetrate through the SWBNNT. The process takes place only when the hydrogen having kinetic energy of above $14 \mathrm{eV}$ impinges vertically at the center of a $\mathrm{BN}$ ring in the tube, which means that the process is impossible to occur in nature. From all the simulation results mentioned so far, no physisorption behavior of hydrogen is observed. Probably, the physisorption energy of a hydrogen molecule is very low and thus the physisorbed hydrogen is released at the simulation temperature of $300 \mathrm{~K}$.

The trapping mechanism of a hydrogen molecule with the incident energy of $26 \mathrm{eV}$, case (iii) shown in Fig. 2, is illustrated in Fig. 4(a) through time evolution of the kinetic energy for two hydrogen atoms indexed by $\mathrm{H} 1$ and $\mathrm{H} 2$ in a $\mathrm{H}_{2}$ molecule up to $1000 \mathrm{MD}$ steps. Also, the inset shows the detailed penetration behavior of the hydrogen atoms into the tube. Here, $\mathrm{H} 1$ means a front hydrogen atom when a hydrogen molecule moves to the SWBNNT and H2 is a rear one. On moving to the BNNT, a $\mathrm{H} 1$ atom has a kinetic energy of about $27.5 \mathrm{eV}$ which is $1.5 \mathrm{eV}$ higher than the initial incident energy, $26 \mathrm{eV}$, of a $\mathrm{H}_{2}$ molecule. This is caused by the trans- fer of kinetic energy of a $\mathrm{H} 2$ atom, which can be deduced from the fact that the kinetic energy of $\mathrm{H} 2$ decreases to $23.5 \mathrm{eV}, 1.5 \mathrm{eV}$ lower than the incident energy. At 13 steps, the kinetic energy of the $\mathrm{H} 1$ atom is lowered to a local minimum, and then the $\mathrm{H} 1$ atom enters the tube. The $\mathrm{H} 1$ atom loses about $16 \mathrm{eV}$ during this penetration process. However, the kinetic energy of $\mathrm{H} 1$ rises quickly to $24 \mathrm{eV}$ on entering with the high repulsive force exerted on $\mathrm{H} 1$ by the tube wall. This force results from the electron overlap between a $\mathrm{H} 1$ atom and $\mathrm{B}$ or $\mathrm{N}$ atom of the nanotube when $\mathrm{H} 1$ is passing through the tube wall. Because of a limited space in the tube, the encapsulated hydrogen atom frequently collides with the
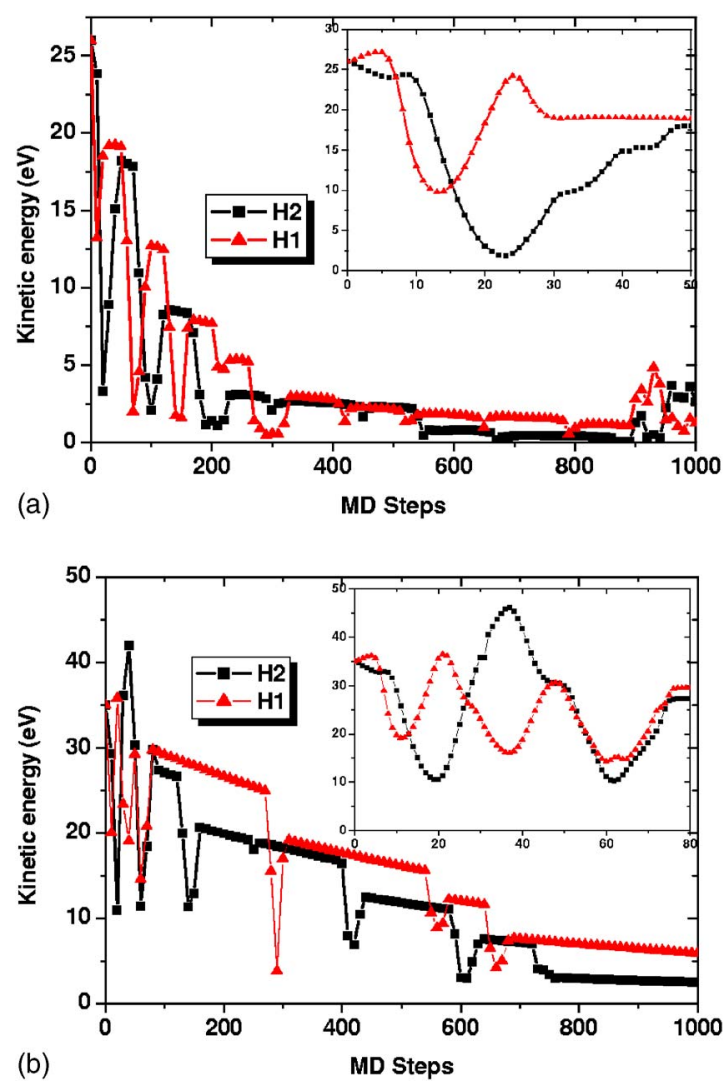

FIG. 4. Time evolution of the kinetic energy of a hydrogen molecule colliding with a $(10,0)$ SWBNNT at the incident energies of (a) 26 and (b) $35 \mathrm{eV}$. $\mathrm{H} 1$ and $\mathrm{H} 2$ denote front and rear hydrogen atoms when the $\mathrm{H}_{2}$ molecule penetrates into the tube. (a) is the same case with Fig. 2 and (b) is for Fig. 3. The insets show the penetration and escaping mechanism of a hydrogen molecule in detail. 
TABLE II. Behaviors of a hydrogen atom impinging with its incident direction and kinetic energy. Here modes (A), (B), (C), and (D) are similar to those of Fig. 1.

\begin{tabular}{|c|c|c|}
\hline & $\begin{array}{l}\text { Incident energy } \\
\text { range }(\mathrm{eV})\end{array}$ & Phenomena \\
\hline \multirow[t]{3}{*}{ Mode (A) } & $1-21$ & A hydrogen molecule rebounds off the sidewall of the SWBNNT. \\
\hline & $22-27$ & A hydrogen atom penetrates through the wall, and then is trapped inside the tube. \\
\hline & $28-35$ & A hydrogen atom enters the tube but escapes through the other side. \\
\hline \multirow[t]{3}{*}{ Mode (B) } & $1-32$ & A hydrogen atom rebounds off the sidewall of the SWBNNT. \\
\hline & $33-34$ & A hydrogen atom penetrates through the wall, and then is trapped inside the tube. \\
\hline & 35 & A hydrogen atom enters the tube but escapes through the other side. \\
\hline \multirow[t]{4}{*}{ Mode $(C)$} & $1-4$ & A hydrogen atom rebounds off the sidewall of the tube. \\
\hline & $5-7$ & A hydrogen atom is chemisorbed by the impacted B atom. \\
\hline & $8-9$ & $\begin{array}{l}\text { A hydrogen atom is physisorbed on the wall after temporary B chemisorption } \\
\text { with the impacted B atom. }\end{array}$ \\
\hline & $10-35$ & A hydrogen atom rebounds off the sidewall. \\
\hline Mode $(D)$ & $1-35$ & A hydrogen atom rebounds off the sidewall. \\
\hline
\end{tabular}

tube wall, which results in decreasing kinetic energy in Fig. 4(a). After the penetration, the $\mathrm{H} 1$ atom collides with the tube at first at 25 steps and then the kinetic energy of $\mathrm{H} 1$ decreases gradually, indicating that most of it is transferred to the nanotube. After the 25 steps, the $\mathrm{H} 1$ atom flies without any disturbance until 50 steps. In the case of $\mathrm{H} 2$, the kinetic energy is lowered to the first local minimum at 23 steps and the $\mathrm{H} 2$ enters the tube, followed by the loss of kinetic energy of $23 \mathrm{eV}$. Then, until 50 steps, the kinetic energy of the $\mathrm{H} 2$ atom recovers gradually to about $18 \mathrm{eV}$. After 50 steps, both of the $\mathrm{H} 1$ and $\mathrm{H} 2$ atoms having the kinetic energies of $18 \mathrm{eV}$ collide with the tube for several times, which causes oscillation of the kinetic energies. The collision process makes the kinetic energy decrease gradually. At 900 steps, the kinetic energies of the $\mathrm{H} 1$ and $\mathrm{H} 2$ indicate 2.6 and $1.2 \mathrm{eV}$, respectively. It is also found that the kinetic energies for both atoms are abruptly increased near 900 steps, because the $\mathrm{H} 1$ and $\mathrm{H} 2$ atoms reform a hydrogen molecule. The reaction, $\mathrm{H}+\mathrm{H}$ $\rightarrow \mathrm{H}_{2}$, is exothermic, resulting in the increase of system temperature, therefore velocities of $\mathrm{H} 1$ and $\mathrm{H} 2$ are increased. The flattops shown in Fig. 4(a) correspond to the free-fly periods from the collision to the next inside the tube.

The evolution of kinetic energy of two hydrogen atoms ( $\mathrm{H} 1$ and $\mathrm{H} 2$ ) in a $\mathrm{H}_{2}$ molecule is shown in Fig. 4(b) with MD time steps. The incident energy was $35 \mathrm{eV}$, case (vi) as in Fig. 3, and both hydrogen atoms are expected to escape from encapsulation in the tube. Similar to the case of Fig. 4(a), both hydrogen atoms at first lose most energy on arriving at the tube. They are used up to overcome the potential barrier of the tube wall and thus they are decreased significantly. After colliding with the tube wall, the $\mathrm{H}_{2}$ molecule is dissociated into two atoms. Penetration of $\mathrm{H} 1$ and $\mathrm{H} 2$ occurs at 11 and 20 steps, respectively. The kinetic energy of $\mathrm{H} 1$ abruptly increases to about $36 \mathrm{eV}$, which is similar to the initial incident energy of $25 \mathrm{eV}$. This occurs because of the repulsive force exerted on $\mathrm{H} 1$ by the tube wall and the transfer of the kinetic energy from H2. From 11 to 37 steps, the kinetic energy of the $\mathrm{H} 1$ is decreased. In the case of a $\mathrm{H} 2$ atom, its kinetic energy increases to $46 \mathrm{eV}$ at 37 steps, larger than the initial value. After then, due to the transfer of the kinetic energy from $\mathrm{H} 2$ to $\mathrm{H} 1$, the kinetic energy of the $\mathrm{H} 1$ atom is increased, while that of the $\mathrm{H} 2$ is decreased to 50 steps in which both atoms almost simultaneously escape through the opposite tube wall. After the escape of the hydrogen atom, we find several flattops of their kinetic energies which correspond to the free-fly periods from the collision of the hydrogen atom upon the external wall of the tube to the next collision.

Until now, we investigate collision behaviors of a hydrogen molecule with the SWBNNT as a function of its incident direction and kinetic energy. The behaviors for a hydrogen atom are summarized in Table II. The lowest incident energy needed for a hydrogen atom to penetrate a hexagon ring on the nanotube is estimated around $22 \mathrm{eV}$ which is higher than the case $(14 \mathrm{eV})$ of a hydrogen molecule. We think that the front atom (H1) in a hydrogen molecule has kinetic energy of about $22 \mathrm{eV}$ before penetration into the tube after collision with the tube wall because of transfer of kinetic energy of the rear atom (H2) although the initial kinetic energy of the hydrogen molecule is $14 \mathrm{eV}$. This transfer of kinetic energy can be found in Fig. 4. A hydrogen molecule is able to enter the tube only if the incident direction is vertical to the hexagon ring on the tube [mode (A)]. However, a hydrogen atom can do that when the incident direction is $45^{\circ}$ to the wall [mode (B)] if the kinetic energy is very high, more than $33 \mathrm{eV}$, not only when the direction is vertical to the hexagon [mode (A)]. In mode (C), chemisorption and physisorption properties of a hydrogen atom are found in the energy ranges of 5-7 and 8-9 eV, respectively. Above $10 \mathrm{eV}$, however, the hydrogen atom rebounds off the sidewall. In the case of mode (D) a hydrogen atom always rebounds off the sidewall irrespective of the incident energies, different from mode (C).

As discussed above, it needs about 14 and $22 \mathrm{eV}$ each for a $\mathrm{H}_{2}$ molecule and a $\mathrm{H}$ atom to penetrate the wall of a SWBNNT, implying that it is not easy to observe the process in real experiments because the energy is very high. To describe more generally the interaction process of the 

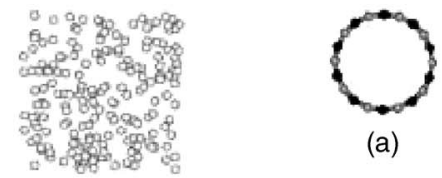

(a)

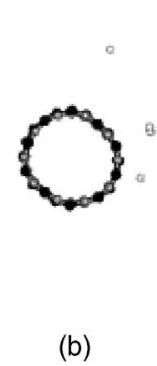

(b)
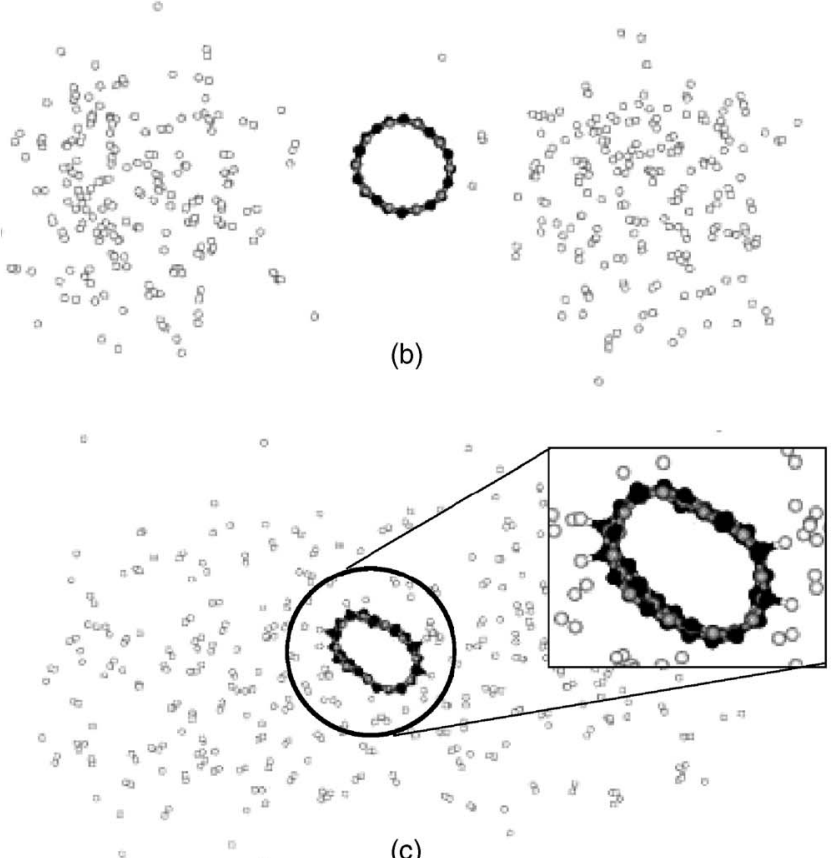

(c)

FIG. 5. Snapshots of interaction between a $(10,0)$ SWBNNT and hydrogen gas consisting of 200 hydrogen atoms, taken at $300 \mathrm{~K}$ at simulation times of (a) 0 , (b) 1.25 , and (c) $12.50 \mathrm{ps}$.

SWBNNT with a hydrogen gas and hydrogen atoms we performed the simulation outlined in Fig. 5. The simulation box size is $60 \times 20 \times 13.14 \AA^{3}$ and both ends of the simulation box outside the nanotube are occupied with dense hydrogen gas consisting of 200 hydrogen atoms each. During the simulation, which was performed at $300 \mathrm{~K}$ under canonical ensemble conditions, most of the hydrogen atoms recombine into $\mathrm{H}_{2}$ molecules. With increased simulation time, most of $\mathrm{H}_{2} / \mathrm{H}$ gas diffuses towards the nanotube and the remaining $\mathrm{H}$ radicals react to form several $\mathrm{B}-\mathrm{H}$ bonds [Fig. 5(c)]. The hydrogen molecules do not react with the nanotube. B-H bonds are created more frequently than the $\mathrm{N}-\mathrm{H}$ bonds, which is in agreement with the results we described earlier (Table II).

To investigate the chemical dissociation of a hydrogen molecule on the SWBNNT wall, we consider the reaction from physisorption of hydrogen to chemisorption on the exterior wall of the $(10,0)$ SWBNNT at room temperature using NVT ensemble MD simulation employing the ReaxFF ${ }_{\mathrm{HBN}}$ (Fig. 6), using restraints to drive the reaction. Similar to a pristine SWCNT, ${ }^{6}$ we find that the reaction from physisorption of a hydrogen molecule into chemisorption on the tube wall is endothermic by about $25 \mathrm{kcal} / \mathrm{mol}$ and the activation energy is very high (about $130 \mathrm{kcal} / \mathrm{mol}$ ). Therefore it is not considered to be thermodynamically or kinetically favorable for the reaction to take place in the room temperature, which makes a serious obstacle of the pristine $(10,0)$ SWBNNT as a hydrogen storage medium.

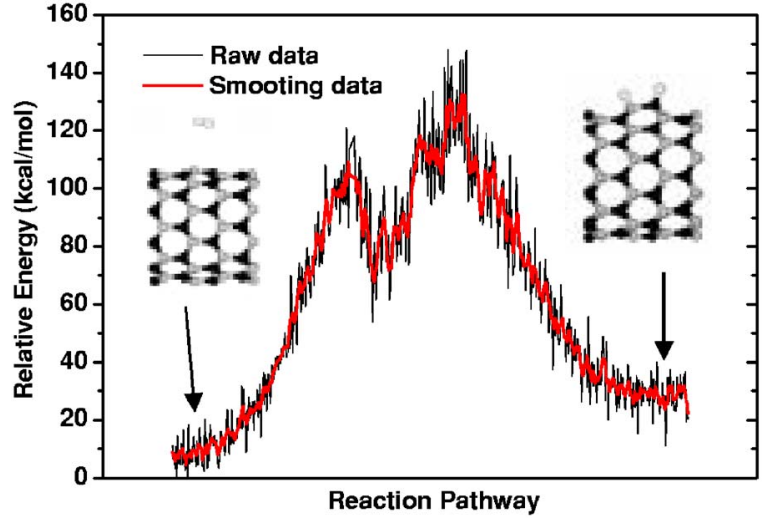

FIG. 6. Reaction pathway for hydrogen from physisorption to chemisorption on the external wall of a SWBNNT, predicted by MD simulation using the ReaxFF $F_{\text {HBN }}$.

\section{B. DFT studies}

Using DFT calculations, we also investigated the optimized structure of $(10,0)$ SWBNNT interacted and bonded with one hydrogen atom. When one hydrogen atom is chemisorbed with one boron atom on the exterior wall of the tube, the length of the $\mathrm{B}-\mathrm{H}$ bond is $1.307 \AA$, slightly larger than that $(1.23 \AA)$ predicted by the ReaxFF ${ }_{\mathrm{HBN}}$ in Fig. 6 . The lengths of $\mathrm{B}-\mathrm{H}$ bond in $\mathrm{BH}_{3}$ (singlet) and $\mathrm{BH}_{4}$ (doublet), calculated with the DFT, is $1.190 \AA$ for the $\mathrm{BH}_{3}$, and 1.182 and $1.288 \AA$ for the $\mathrm{BH}_{4}$, which is shorter than the bond length on the BNNT wall. When one hydrogen atom is chemisorbed with boron, the bonding character of boron is changed to $s p^{3}$ from $s p^{2}$, which results in a change of the $\mathrm{B}-\mathrm{N}$ bond lengths near $\mathrm{B}-\mathrm{H}$. The lengths of three B-N bonds connected with a $\mathrm{B}-\mathrm{H}$ bond are $1.500,1.514$, and $1.514 \AA$, while the $\mathrm{B}-\mathrm{N}$ bond length far from the $\mathrm{B}-\mathrm{H}$ bond is $1.456 \AA$. The angles of $\mathrm{N}-\mathrm{B}-\mathrm{H}$ and $\mathrm{N}-\mathrm{B}-\mathrm{N}$ near a $\mathrm{B}-\mathrm{H}$ bond are $102^{\circ}$ and $114^{\circ}$, respectively.

When one hydrogen atom is chemisorbed with one nitrogen atom, the length of $\mathrm{N}-\mathrm{H}$ is $1.044 \AA$ which is shorter than that of $1.090 \AA$ predicted by the ReaxFF ${ }_{\mathrm{HBN}}$ (see Fig. 6). The $\mathrm{N}-\mathrm{H}$ bond lengths in $\mathrm{NH}_{3}$ (singlet) and $\mathrm{NH}_{4}$ (doublet), calculated with DFT, are 1.016 and $1.062 \AA$ A Hence the $\mathrm{N}-\mathrm{H}$ bond length on the BNNT wall is shorter than those in $\mathrm{NH}_{3}$ and $\mathrm{NH}_{4}$. The lengths of three $\mathrm{B}-\mathrm{N}$ bonds connected with $\mathrm{N}-\mathrm{H}$ are $1.548,1.548$, and $1.540 \AA$ while the $\mathrm{B}-\mathrm{N}$ bond length far from the $\mathrm{B}-\mathrm{H}$ bond is $1.456 \AA$. The angles of $\mathrm{B}-\mathrm{N}-\mathrm{H}$ and $\mathrm{B}-\mathrm{N}-\mathrm{B}$ near the $\mathrm{N}-\mathrm{H}$ bond are about $114^{\circ}$ and $94^{\circ}$. The binding energy of $\mathrm{B}-\mathrm{H}$ and $\mathrm{N}-\mathrm{H}$ bonds on the SWBNNT is also calculated. The binding energy, $\Delta E_{\text {bind }}$, is defined as follows:

$$
\Delta E_{\mathrm{bind}}=E_{\mathrm{BN}+\mathrm{H}}-E_{\mathrm{BN}}-E_{\mathrm{H}},
$$

where $E_{\mathrm{BN}+\mathrm{H}}, E_{\mathrm{BN}}$, and $E_{\mathrm{H}}$ are the total energies of the fully optimized $\mathrm{BN}-\mathrm{H}$ structure, the nanotube alone, and the hydrogen atom, respectively. According to this definition, a stable system is supposed to have a negative binding energy. The binding energies of $\mathrm{B}-\mathrm{H}$ and $\mathrm{N}-\mathrm{H}$ on the exterior wall of the tube are obtained as -8.095 and $7.249 \mathrm{kcal} / \mathrm{mol}$ which are similar with those reported in previous work. ${ }^{26}$ The ReaxFF $F_{\mathrm{HBN}}$ predicts -16.143 and $3.947 \mathrm{kcal} / \mathrm{mol}$ for the 


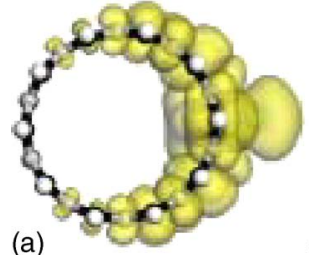

(b)
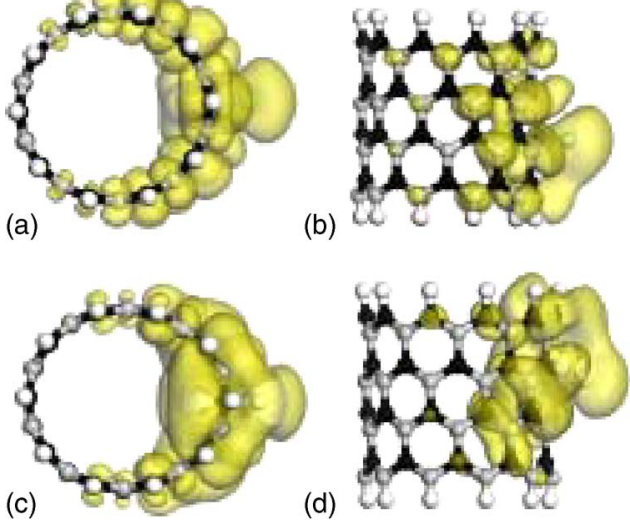

FIG. 7. Distributions of spin density in the optimized (10,0) SWBNNT: (a) and (b) show the cases where hydrogen is chemisorbed upon a boron atom in the nanotube while (c) and (d) show the cases where hydrogen is chemisorbed upon a nitrogen atom. (a) and (c) are plan views while (b) and (d) are side views.

binding energies of the $\mathrm{B}-\mathrm{H}$ and $\mathrm{N}-\mathrm{H}$ on the exterior nanotube wall. Since the $\mathrm{N}-\mathrm{H}$ bond has a positive binding energy, it is difficult to take place. This is in agreement with our ReaxFF $F_{\mathrm{HBN}}$ results in Fig. 5, where we found that hydrogen adsorbed upon the exterior tube wall favors $\mathrm{B}-\mathrm{H}$ bond formation over $\mathrm{N}-\mathrm{H}$ bond formation. Moreover, the $\mathrm{C}-\mathrm{H}$ binding energy in the SWCNT is about $-45 \mathrm{kcal} / \mathrm{mol},{ }^{6}$ much higher than the $\mathrm{B}-\mathrm{H}$ binding energy $(-8.095 \mathrm{kcal} / \mathrm{mol})$ in the BNNT. As such, we expect that hydrogen adsorbed on the BNNT wall can be desorbed at lower temperatures than in the case of a CNT. We also calculate the binding energies of $\mathrm{B}-\mathrm{H}$ and $\mathrm{N}-\mathrm{H}$ on the interior wall of the tube, which are 1.515 and $27.974 \mathrm{kcal} / \mathrm{mol}$, while 3.148 and $5.498 \mathrm{kcal} / \mathrm{mol}$ by ReaxFF $F_{\mathrm{HBN}}$. From the fact that both of the $\mathrm{B}-\mathrm{H}$ and $\mathrm{N}-\mathrm{H}$ bonds are positive binding energy, it is found that the chemisorption of hydrogen atoms inside the tube is thermodynamically unlikely, which is in agreement with the MD results in Figs. 2 and 3.

In order to investigate the distribution of electrons in the optimized BNNT, the spin density of each structure is computed. The spin charge density means the difference between the electron density for alpha-spin and beta-spin electrons. When $\mathrm{H}$ is chemisorbed with a boron atom in Fig. 7(a), most of the charge remains localized on the nanotube wall near a $\mathrm{B}-\mathrm{H}$ bond and a little charge is distributed over $\mathrm{N}$ atoms. In particular, there is no charge distribution around the opposite side of the tube wall (the side without a B-H bond). Similarly, when $\mathrm{H}$ is chemisorbed with a nitrogen atom in Fig. 7(b), much of the charge density is distributed over the tube wall near a $\mathrm{N}-\mathrm{H}$ bond and the charge density is not observed around the other side of the wall. Only contrary to the case of $\mathrm{B}-\mathrm{H}$, a little charge is distributed over B atoms.

\section{CONCLUSIONS}

From our ReaxFF $F_{\mathrm{HBN}}$ MD simulations and DFT calculations, the ReaxFF $F_{\mathrm{HBN}}$ developed in the previous work, Part I, can appropriately describe the interaction of hydrogen with BNNTs. We found that the kinetic energy of $14 \mathrm{eV}$ is required for the $\mathrm{H}_{2}$ molecule to penetrate through the wall of the BNNT. In addition chemical dissociation of $\mathrm{H}_{2}$ upon the nanotube at room temperature is also found to be thermodynamically unfavorable. On the other hand, we predict that $\mathrm{H}$ atoms are able to chemically adsorb on the exterior wall of the nanotube and prefer to make $\mathrm{B}-\mathrm{H}$ bonds over $\mathrm{N}-\mathrm{H}$ bonds. These results indicate that hydrogen storage is very difficult through chemical binding to BNNTs or diffusion to the interior of the BNNT. Therefore, we consider that future efforts to apply BNNTs for hydrogen storage should be focused on other treatments of the BNNT such as doping on its surfaces by other elements or generation of nanopores to increase its hydrogen storage capacity and change of heat in hydrogen adsorption.

\section{ACKNOWLEDGMENTS}

This research was supported by a grant (Code No. 04K1501-02210) from "Center for Nanostructured Materials Technology" under "21st Century Frontier R\&D Programs" of the Ministry of Science and Technology, Korea.

${ }^{1}$ A. C. Dillon, K. M. Jones, T. A. Bekkedahl, C. H. Klang, D. S. Bethune, and M. J. Heben, Nature (London) 386, 377 (1997).

${ }^{2}$ Q. Wang and J. K. Johnson, J. Chem. Phys. 110, 577 (1999).

${ }^{3}$ R. T. Yang, Carbon 38, 623 (2000).

${ }^{4}$ E.-C. Lee, Y.-S. Kim, Y.-G. Jin, and K. J. Chang, Phys. Rev. B 66, 073415 (2002).

${ }^{5}$ J. Li, T. Furuta, H. Goto, T. Ohsashi, Y. Fujiwara, and S. Yip, J. Chem. Phys. 119, 2376 (2003).

${ }^{6}$ S. S. Han and H. M. Lee, Carbon 42, 2169 (2004).

${ }^{7}$ N. G. Chopra, R. J. Luyken, K. Cherrey, V. H. Crespi, M. L. Cohen, S. G. Louie, and A. Zettl, Science 269, 966 (1995).

${ }^{8}$ H. J. Xiang, J. Yang, J. G. Hou, and Q. Zhu, Phys. Rev. B 68, 035427 (2003).

${ }^{9}$ S. W. Yang, H. Zhang, J. M. Soon, C. W. Lim, P. Wu, and K. P. Loh, Diamond Relat. Mater. 12, 1194 (2003).

${ }^{10}$ I. Narita and T. Oku, Diamond Relat. Mater. 11, 945 (2002).

${ }^{11}$ T. Oku, M. Kuno, and I. Narita, J. Phys. Chem. Solids 65, 549 (2004).

${ }^{12}$ R. Ma, Y. Bando, H. Zhu, T. Sato, C. Xu, and D. Wu, J. Am. Chem. Soc. 124, 7672 (2002).

${ }^{13}$ C. Tang, Y. Bando, X. Ding, S. Qi, and D. Golberg, J. Am. Chem. Soc. 124, 14550 (2002).

${ }^{14}$ J. S. Binkley, J. A. Pople, and W. J. Hehre, J. Am. Chem. Soc. 102, 939 (1980).

${ }^{15}$ C. C. J. Roothan, Rev. Mod. Phys. 23, 69 (1951).

${ }^{16}$ Y. Ma, Y. Xia, M. Zhao, R. Wang, and L. Mei, Phys. Rev. B 63, 115422 (2001).

${ }^{17}$ A. Loiseau, F. Willaime, N. Demoncy, G. Hug, and H. Pascard, Phys. Rev. Lett. 76, 4737 (1996).

${ }^{18}$ E. Bengu and L. D. Marks, Phys. Rev. Lett. 86, 2385 (2001).

${ }^{19}$ M. Terauchi, M. Tanaka, K. Suzuki, A. Ogino, and K. Kimura, Chem. Phys. Lett. 324, 359 (2000).

${ }^{20}$ J. M. Haile, Molecular Dynamics Simulation: Elementary Methods (Wiley, Toronto, 1992).

${ }^{21}$ B. Delley, J. Chem. Phys. 113, 7756 (2000); $\mathrm{DMOL}^{3}$ is a registered software product of Molecular Simulations, Inc.

${ }^{22}$ J. P. Perdew and Y. Wang, Phys. Rev. B 45, 13244 (1992).

${ }^{23}$ A. D. Becke, J. Chem. Phys. 104, 1040 (1996).

${ }^{24}$ W. J. Hehre, L. Radom, P. v. R. Schleyer, and J. A. Pople, Ab Initio Molecular Orbital Theory (Wiley, Toronto, 1986).

${ }^{25}$ B. Delley, J. Chem. Phys. 92, 508 (1990).

${ }^{26}$ X. Wu, J. Yang, J. G. Hou, and Q. Zhu, Phys. Rev. B 69, 153411 (2004). 